\title{
El contexto geopolítico de la explotación forestal en la Península de Yucatán, México ${ }^{1}$
}

\section{THE GEOPOLITICAL CONTEXT OF FOREST EXPLOITATION IN THE YUCATAN PENINSULA, MEXICO}

\author{
O CONTEXTO GEOPOLÍTICO DA EXPLORAÇÃO FLORESTAL NA PENÍNSULA \\ DE YUCATÁN, MÉXICO
}

Para citar este artículo: Alonso Velasco, I. y Velázquez Torres, D. (2019)

El contexto geopolítico de la explotación forestal en la Península de Yucatán, México. Perspectiva Geográfica, 24(1), 116-137. https://doi.org/10.19053/01233769.8427
Ignacio Alonso Velasco ${ }^{2}$

David Velázquez Torres ${ }^{3}$

\section{Resumen}

Este artículo revisa el marco histórico de la explotación forestal y de la pérdida de la riqueza maderera y de la cobertura forestal en relación con el poblamiento rural propiciado por algunas políticas de colonización en la Península de Yucatán desde la década de 1950, como el Programa Nacional de Desmontes, o proyectos económicos como el arrocero, el azucarero y el ganadero. También se analizan los esfuerzos que actualmente se están llevando a cabo para mitigar e, incluso, revertir la deforestación con base en políticas públicas internacionales que han sido asumidas por las auto-

1 El presente artículo se deriva de la tesis doctoral titulada Disputa territorial y coordinación intermunicipal como modelo de gestión pública: Campeche y Quintana Roo, desarrollada con financiamiento de una beca otorgada por el Consejo Nacional de Ciencia y Tecnología (Conacyt). Una versión preliminar de este artículo fue presentada en el VII Simposio Mexicano Colombiano de Investigación Geográfica en Chetumal, México

2 Profesor por asignatura en la Universidad de Quintana Roo, donde se encuentra cursando sus estudios en el Doctorado en Geografía. velasco@uqroo.edu.mx

3 Profesor investigador de tiempo completo en la Universidad de Quintana Roo. Doctor en Geografía por la Universidad Nacional Autónoma de México. davvelaz@uqroo.edu.mx

\section{Recepción:}

18 de enero de 2018

Evaluación:

14 de diciembre de 2018

Aprobación:

25 de febrero de 2019 
ridades mexicanas. Este trabajo cualitativo es producto de la revisión de literatura académica e institucional. Permite advertir cómo el paso de la explotación forestal extractiva a la sustentable aún se sitúa en un contexto en el que la geopolítica es determinante.

Palabras clave: bosques; cambio climático; deforestación; madera; Península de Yucatán.

\section{Abstract}

This article reviews the historical framework of logging and the loss of timber wealth and forest cover in relation to a rural settlement process caused by some colonization policies in the Yucatan Peninsula since the 1950s, such as the National Felling Program and some rice, sugar and livestock production projects. Efforts being made to mitigate and even reverse deforestation following international public policies that have been adopted by Mexican authorities are also discussed. This qualitative paper derives from a review of academic and institutional literature. It is concluded that the transition from extractive to sustainable logging is still placed in a context in which geopolitics is a determining factor.

Keywords: forests; climate change; deforestation; wood; Yucatan Peninsula.

\section{Resumo}

Este artigo revê o marco histórico da exploração florestal e da perda de riqueza madeireira e da cobertura florestal em relação à população rural causada por algumas políticas de colonização na Península de Iucatã desde a década de 1950, como o Programa Nacional de Desmantelamento, ou projetos econômicos como o arrozeiro, o açucareiro e o pecuário. Também se analisaram os esforços que atualmente são realizados para mitigar e até reverter o desmatamento com base em políticas públicas internacionais que têm sido empreendidas pelas autoridades mexicanas. Este trabalho qualitativo é o produto da revisão da literatura acadêmica e institucional. Permite observar como o caminho da exploração florestal extrativa em direção à sustentabilidade ainda está em um contexto em que a geopolítica é decisiva.

Palavras chave: florestas; mudança climática; desmatamento; madeira; Península de Yucatán 


\section{Introducción}

No se puede hablar de la Península de Yucatán sin hacer referencia a su mayor riqueza, la forestal. Esta ha sido la razón principal de una disputa territorial que inició en 1902, cuando se creó el Territorio Federal de Quintana Roo por medio de un decreto presidencial de Porfirio Díaz (Ramayo, 1997). En ese documento se realizó una deficiente delimitación del nuevo territorio, lo cual ha dado pie a que las autoridades yucatecas y campechanas no hayan dejado de pugnar por seguir disfrutando de los recursos forestales de un espacio bajo indefinición jurisdiccional.

La Península de Yucatán es una región que alberga una gran diversidad de flora y fauna que ha sido una gran fuente de recursos naturales, los cuales se erigen sobre un substrato kárstico. La presencia de rocas calizas hace que el suelo sea muy poroso y haya una rápida filtración del agua, lo que, en principio, convertiría al terreno en infértil y poco productivo. Sin embargo, la presencia de un rango elevado de 180 a 270 días de humedad al año ${ }^{4}$ y el predominio de los tipos de suelo denominados Vertisol $^{5}$ y Phaeozem ${ }^{6}$ propician la existencia de toda esa masa forestal que ha habido de forma ancestral en la península yucateca.

$4 \quad$ Este rango de humedad se denomina ústico según el sistema de clasificación climática Thornwaite, el cual favorece la presencia de agricultura y ganadería en la zona.

5 Son suelos de climas templados y cálidos, especialmente de zonas con una marcada estación seca y otra lluviosa. Se caracterizan por su estructura masiva y su alto contenido de arcilla. Su uso agrícola es muy extenso, variado y productivo. Son muy fértiles, pero su dureza dificulta la labranza.

6 Se pueden presentar en cualquier tipo de relieve y clima. Se caracterizan por tener una capa superficial oscura, suave, rica en materia orgánica y en nutrientes. Son de profundidad muy variable. El uso óptimo de estos suelos depende en muchas ocasiones de otras características del terreno y, sobre todo, de la disponibilidad del agua para riego.
Desde el siglo XVI, el modelo de explotación forestal fue el precursor de la actividad económica. La extracción del palo de tinte (Haematoxylum campechianum) se realizó en los siglos XVII y XVIII, y la del látex de chicozapote (Manilkara zapota) desde finales del XIX y hasta mitad del XX; maderas preciosas como la caoba (Swietenia macrophylla) y cedro (Cedrela odorata) fueron explotadas de la década de los cuarenta a los ochenta. A partir 1978, ha habido problemas de deforestación en la región (Amor-Conde, Colchero, Rivera y Manterola, 2005) debido al crecimiento poblacional y a las consecuentes demandas de los pobladores por el cambio del uso de suelo forestal a campos agrícolas y ganaderos. Incluso un espacio protegido como la Reserva de la Biosfera Calakmul es considerado bajo la categoría de "amenazado críticamente", por lo que se requiere tomar medidas inmediatas para evitar que fracase en la protección y el mantenimiento de la diversidad biológica. Un número importante de amenazas son las originadas por los seres humanos, como incendios forestales, tala ilegal, deforestación, cacería de fauna silvestre y carreteras para los grandes proyectos turísticos, como Mundo Maya y las Unidades de Manejo y Aprovechamiento Sustentable de Vida Silvestre (UMA).

Este artículo examina el marco histórico de la explotación de esta riqueza maderera y de la pérdida de la cobertura forestal en relación con el poblamiento rural propiciado por las instituciones públicas. Asimismo, se estudian los esfuerzos que actualmente se están llevando a cabo para mitigar e, incluso, revertir la deforestación con base en políticas públicas internacionales que han sido asumidas por las autoridades mexicanas. 


\section{Marco histórico de la}

\section{industria de la madera y el chicle}

\section{en la Península de Yucatán}

Los mayas llevaban a cabo una agricultura de tumba-roza y quema y contaban con un sistema denominado en lengua maya T'olché, que consistía en dejar áreas extensas tan solo para la recuperación de la tierra, las cuales fungían además como barreras de protección (Ferré, 2001). Con la Colonia comenzó la explotación del palo de tinte. La baja densidad demográfica de la Península de Yucatán y la política de represión aplicada a los mayas se conjugaron para considerar como un espacio vacío los extensos territorios de la costa oriental. Una vez extraído el palo de tinte o de Campeche, se transportaba hasta Inglaterra. El colorante que se obtenía de esta madera era muy apreciado por la industria textil inglesa para teñir de negro, azul y púrpura, la lana, el lino, el algodón y los sombreros.

Las áreas de mayor extracción de palo de tinte se encontraban en el centro de Quintana Roo y hacia el sur, donde solo existía la población de Bacalar. La decadencia del comercio del palo de tinte se dio a fines del siglo XIX cuando el inglés Perkin desarrolló el primer pigmento textil en el año de 1868, lo que permitió la fabricación de anilinas sintéticas que acabaron por sustituir el uso de colorantes naturales e hicieron innecesaria la adquisición del producto que durante dos siglo y medio había sostenido la vida económica de la región. A principios del siglo XX, el desplome de la producción y la exportación del palo de tinte era total (Leriche, 2001).

No obstante, la decadencia gradual de esta mercancía se compensó con la creciente exportación de maderas preciosas, como la caoba y el cedro, y poco después, al despuntar el siglo XX, el chicle extraído del árbol del zapote se sumó a los productos exportados. En 1860 inició la historia comercial del árbol del chicle como goma, lo cual incrementa el interés en la región.

La constitución de un nuevo territorio, como espacio geopolítico y de control administrativo en la costa oriental de Yucatán, fue una propuesta que presentó Sierra Méndez ${ }^{7}$ al general Díaz ${ }^{8}$ en junio de 1896 como reacción provocada por la lucha de intereses regionales e internacionales sobre un espacio que no lograban controlar las autoridades yucatecas, pero que era domesticado por el poder militar indígena con apoyo de la Corona inglesa por medio de sus relaciones con Honduras Británica (Quintal y Chan, 2011). Era necesario controlar la frontera con Belice para poder proceder a la explotación forestal por medio de concesiones y evitar la fuga de dichos recursos por el cauce del río Hondo a favor de la colonia británica. Esta misión fue encomendada por el presidente Díaz al teniente Othón P. Blanco Núñez ${ }^{9}$, quien instaló una aduana flotante en la desembocadura de dicha corriente fluvial, a bordo de un pontón. Esto no era

7 Justo Sierra Méndez (San Francisco de Campeche, 1948-Madrid, 1912) fue escritor, historiador, periodista, poeta, político y filósofo. Fue promotor de la fundación de la actual Universidad Nacional Autónoma de México (UNAM). Es considerado uno de los personajes más influyentes de la historia moderna de México. Conocido como Maestro de América, por el título que le otorgaron varias universidades de América Latina. En su honor se le puso su nombre a una de las localidades de la zona objeto de estudio, la cual fue encuestada en este trabajo de investigación.

8 José de la Cruz Porfirio Díaz Mori (Oaxaca de Juárez, 1830-París, 1915) fue un militar mexicano que ejerció el cargo de presidente de México en siete ocasiones, durante 30 años. Convencido defensor del progreso, entre los principales logros de su mandato se encuentra la expansión del ferrocarril en México.

9 Othón Pompeyo Blanco Núñez de Cáceres (Ciudad Victoria, 1868-Ciudad de México, 1959) fue un destacado marino mexicano, fundador de Payo Obispo (actual ciudad de Chetumal) el 5 de mayo de 1898, lugar en donde se encuentran sus restos. Llegó a ser vicealmirante tras 61 años de servicio. 
suficiente, sino que se hacía imperante también poder ocupar los terrenos habitados por los mayas, lo cual se logró por medio de una ofensiva militar en 1901, que forzó la caída del principal reducto de los indígenas sublevados, Chan Santa Cruz, ante las tropas federales.

Este hecho abrió mejores expectativas a la ocupación del territorio oriental por las compañías deslindadoras y por los concesionarios forestales. En los años de 1911 y 1912, al menos diez concesionarios de las selvas se dividían y disputaban lo que ahora es el Estado de Quintana Roo. Los mayas perdieron la mayoría de sus tierras a favor de grandes empresas de explotación forestal estrechamente asociadas al mercado mundial (Bracamonte y Sosa, 2000).

En aquel entonces operaban en territorio campechano ocho grandes empresas madereras que en total poseían alrededor de dos millones de hectáreas, casi la quinta parte de las tierras de Campeche. Una sola compañía, la Laguna Corporation, representante de la Wrigley and Co., usufructuaba $7.600 \mathrm{~km}^{2}$ y contaba con importantes instalaciones que incluían vías de ferrocarril, líneas telefónicas, talleres y bodegas (Leriche, 2001). También destaca en los años veinte la American Chicle. Esas empresas tenían ramificaciones en Payo Obispo, Campeche, Mérida, Ciudad de México, Belice y Guatemala. Aunque fueron las más importantes, había otras compañías menores con intereses en la Península de Yucatán, como la Casa Bromgber o la Negociación Chiclera Mexicana (Ramos, 1999).

Al no estar claramente definidos los límites territoriales de Quintana Roo, los estados vecinos extendían su acción más allá de su jurisdicción política. Las concesiones forestales son un buen ejemplo de ello. Los gobiernos de Campeche y Yucatán otor- gaban permisos de corte de madera o extracción de chicle en las zonas colindantes con el territorio. Cuando surgía alguna dificultad en dichas concesiones, entraban en acción los representantes de esos gobiernos, las autoridades del territorio o las nacionales (Higuera, 1997). Uno de los casos más representativos de la época, por sus alcances y su duración, fue la autorización otorgada al libanés Antonio Soda, quien extraía chicle al suroeste de Quintana Roo, en las cercanías del poblado de Icaiché. En 1912 este permisionario fue acusado por los pobladores mayas de talar los bosques de su concesión y de realizar explotación fraudulenta de la selva circundante a dicha comunidad. Las autoridades quintanarroenses, al estar sujetas a las decisiones de las federales, no pudieron ejercer una acción inmediata. En 1913, a más de nueve meses de iniciado el conflicto, aún no se podía enviar a la comisión del gobierno territorial para que solucionase las diferencias entre los mayas y el concesionario.

Este ejemplo también sirve para ilustrar que la riqueza de las selvas en las que habitaban los mayas solo pasó por sus manos. Las ganancias iban a parar a las arcas de los intermediarios que vendían la goma a las grandes compañías de los Estados Unidos (Ramos, 1999). Con el crecimiento de la industria, empresarios chicleros de Veracruz, Chiapas, Yucatán y Belice llegaron a Quintana Roo en 1915; para esa época, más del 70\% de la goma de mascar que se consumía en Estados Unidos procedía de México.

Años después, durante la Primera Guerra Mundial, la industria del chicle experimentó un auge comercial, ya que el ejército estadounidense lo usaba como sustituto del tabaco a fin de calmar los nervios y mitigar la sed. El chicle era transportado des- 
de los campamentos chicleros hasta los centros de recepción en Santa Cruz de Bravo, Payo Obispo y Cozumel, así como a Valladolid y Peto en Yucatán.

La recesión financiera estadounidense de 1929 provocó un déficit económico en la administración territorial, lo cual fue usado como argumento en la década de 1930 para justificar la extinción jurídica de Quintana Roo como territorio y su anexión a los estados de Yucatán y Campeche. Esto hasta que el presidente Lázaro Cárdenas lo declaró otra vez territorio el 11 de enero de 1935. Un panorama de abandono caracterizaba a los antiguos campamentos y pequeños núcleos de población que se habían fincado en las áreas chicleras y madereras. De acuerdo a los datos censales, en 1940 habían desaparecido 231 núcleos de población registrados en 1920, cuando la explotación de maderas llegó a sus máximos volúmenes. Vastas regiones que fueron boscosas y fértiles quedaron saqueadas, forzando la emigración de alrededor de 15.000 habitantes de esos improvisados poblados. Esta situación cambió cuando la producción de chicle creció notoriamente durante la Segunda Guerra Mundial, hasta alcanzar un máximo histórico de 3.876 toneladas en 1942 .

El día 30 de julio de 1954 se constituyó por medio de un decreto la empresa Maderas Industrializadas de Quintana Roo (Miqro), la cual fue acreedora de una extensión de 4.629 km². Así se comenzó una nueva etapa, en la que se pretendía lograr la recuperación de los bosques en un ambiente de desarrollo silvícola sustentable.

Sin embargo, tan solo un año después, Chetumal y la región sur del territorio sufrió el embate del ciclón Janet la noche del 27 de septiembre de 1955. El meteoro derribó gran parte de la riqueza forestal, por lo que se dejaron de realizar trabajos de Vol. 24 N. ${ }^{\circ} 1$ chicle en la región para dedicarse al rescate de las maderas finas caídas o dañadas, como la caoba.

Esta fue una tarea costosa debido a la multitud de troncos y raíces que dificultaban sacar las caobas, ya que mientras que los trabajadores, en épocas normales, podían caminar sin dificultades en la selva unos ocho o diez kilómetros al día, tras el ciclón Janet no lograban andar más de dos kilómetros en una jornada completa. Por medio del Banco de Comercio Exterior se hicieron los trámites para hacer costeable el rescate de esa operación maderera. En la primera temporada, de 1955-1956, se logró extraer y transformar en trozas y tablas aserradas alrededor de $100.000 \mathrm{~m}^{3}$ de caoba, y en la siguiente temporada (1956-1957), otro tanto igual o más, cuando lo normal era explotar anualmente tan solo unos $15.000 \mathrm{~m}^{3}$ antes del huracán (Hoy, 1998, pp. 73-75).

Si desastroso fue el paso del ciclón, peor fue la política colonizadora llevada a cabo por parte de las autoridades a partir de finales de la década de 1950 . En vez de proceder a reforestar, se llevó a cabo un programa devastador de desmonte del bosque tropical, para lo cual se creó un plan nacional. Esta acción permitió ampliar la frontera agrícola, con la finalidad de llevar a cabo plantaciones de azúcar y arroz (Macías, 2004).

Desde la geopolítica, era pertinente traer a colonos de otras partes de la república en las que hubiera una gran presión social sobre la tierra para trabajar estas nuevas tierras ganadas a la selva y así aprovechar para ocupar "espacios vacíos" en zonas sensibles como las fronterizas. Así es que se desplazaron personas originarias de Jalisco, la Comarca Lagunera, Veracruz, Michoacán y Tabasco, entre otros lugares. 
No obstante, la mayoría de estos proyectos agrícolas fracasaron. Se trató de una política poblacional y agraria antiforestal que destruyó grandes áreas de masa arbórea sin que ello supusiera un incremento considerable de la superficie agrícola. Gran parte de las áreas desmontadas fueron abandonadas y los bosques productivos fueron reemplazados por acahuales improductivos (Velázquez, 2001).

En 1983 concluyó la concesión a la Miqro y hubo que replantearse la política forestal. Por ello se elaboró un Plan Piloto Forestal en Quintana Roo para tratar de lograr la conservación de la selva a largo plazo. Dicho plan dio paso, en 1987, a un Plan Forestal Estatal, con el objetivo de favorecer la organización de los propietarios de los montes y establecer una hacienda forestal estable. En todos los ejidos se establecieron áreas forestales permanentes.

Actualmente es posible seguir extrayendo maderas preciosas como el cedro y la caoba, las cuales tienen como principal mercado de exportación el europeo, mientras que a nivel local la industria turística es la principal demandante de estos productos, tal y como señala el Plan Estatal de Desarrollo del Estado de Quintana Roo 2016-2022 (Poder Ejecutivo del Estado de Quintana Roo, 2017). Esta riqueza forestal es la razón principal por la que los estados de Campeche y Quintana Roo litigan la soberanía de una franja de selva de valor económico internacionalmente reconocido.

\section{La relación entre la pérdida de cobertura vegetal y el}

\section{poblamiento rural}

En las últimas cuatro décadas la población mexicana ha crecido de manera considerable, lo cual ha traído consecuencias para el medio ambiente. Esta relación de causa-efecto hace relevante el asunto de la distribución poblacional. La proporción de la población rural del país ha disminuido a través de los años, sin embargo, su tamaño en términos absolutos ha aumentado. De acuerdo con cifras del Instituto Nacional de Estadística y Geografía (Inegi), el número de personas que actualmente habitan en el medio rural de México se incrementó en cerca del $7 \%$ con respecto a 1990, año en el que representaba $28,7 \%$ del total nacional.

Del análisis de la información recabada en fuentes secundarias, se puede evidenciar que las entidades federativas de Campeche y Quintana Roo se convirtieron en entidades receptoras de migrantes desde que, en la última etapa de los años setenta ${ }^{10}$, se gestionaron políticas públicas destinadas a hacer productivo, desde el punto de vista agropecuario, un "espacio vacío" que se hallaba ocupado por selva. Se trató de proyectos económicos como el Programa Nacional de Desmontes, proyectos arroceros y azucareros, o el ganadero en Nicolás Bravo, en los que se confiaba como única posibilidad para poder trasladar, asentar y retener población en un área tan alejada y aislada del resto del país. El repoblamiento se dio en los costados de la carretera federal Escárcega-Chetumal y al sur de la región de Xpujil, y fue realizado mayormente con campesinos que emigraron del centro y este del país (Laguna, 2005), que no habían podido ser ubicados en los márgenes del río Candelaria ni en terrenos de los municipios del Carmen, Champotón y Campeche (Pino, 1997).

10 Las dotaciones anteriores son acciones agrarias aisladas, como es el caso de San Antonio Soda (Gustavo Díaz Ordaz), fundada en 1940, o los ejidos de Nuevo Bécal, 20 de Noviembre y Álvaro Obregón (Zoh-Laguna), que en la década de los sesenta ya se habían creado por resolución presidencial. 
No todos los programas económicos puestos en ejecución tuvieron un desenvolvimiento similar ni igual impacto social y económico territorial. Se destaca el fracaso del programa pecuario de ganadería extensiva (Laguna, 2005) como consecuencia de la dificultad de conseguir agua en esta zona limítrofe, pues se haya a más de 100 metros de profundidad y no había energía eléctrica para extraerla por medio de bombas de combustión interna, o bien se encuentra a menos profundidad, pero no es apta para consumo humano o animal, puesto que contiene partículas de yeso.

También se pretendía con estos proyectos de atracción de población que el Territorio Federal de Quintana Roo se transformara en estado, para lo cual necesitaba contar con las bases económicas necesarias y un mínimo de 80.000 habitantes, cantidad que se logró alcanzar y permitió que, el 8 de octubre de 1974, Quintana Roo naciera como estado libre y soberano.

Para ello no solo se promovieron los programas económicos comentados, sino que también se llevaron a cabo acciones encaminadas al desarrollo de la entidad por medio de la construcción de obras de infraestructura básica: la red de carreteras, la ampliación de los servicios a la población (electrificación de las localidades rurales e impulso a la educación primaria y media, entre otros) y a la producción (obras de irrigación, dotación de tierras y colonización).

Actualmente, las comunidades localizadas dentro de los bosques tropicales de la Península de Yucatán se encuentran alejadas de centros urbanos de rango medio o alto. Según el Consejo Nacional de Evaluación de la Política de Desarrollo Social (Coneval, 2010), el 29,2 \% de la población rural mexicana (12,3 millones de personas) se encuentra en situación de pobreza alimentaria, lo cual afecta su calidad de vida e incrementa su vulnerabilidad. De acuerdo al Consejo Nacional de Población (Conapo, 2012), el 92\% de las localidades rurales mexicanas más aisladas tiene un grado de marginación alto y muy alto. Los espacios productivos de dichas poblaciones están ocupados por tres actividades: la producción de maíz para autoconsumo mediante el uso de la técnica agrícola de roza, tumba y quema, el cultivo comercial del chile jalapeño y la extracción de productos silvestres (García y Pat, 2000).

Álvarez y Herrera (2014) afirman que sí existe relación directa entre la pérdida de cobertura vegetal y el patrón de distribución de los asentamientos rurales, y que las comunidades dispersas desorganizadas, con un tejido social débil, son más proclives a un mal manejo de los recursos naturales en ecosistemas frágiles. De hecho, las regiones ecológicas con mayor número de localidades pequeñas y aisladas son los bosques templados, las selvas húmedas y las selvas secas.

Sobrino, Garrocho, Graizbord, Brambila y Aguilar (2015) refieren que la conversión de terrenos hacia usos agropecuarios es una de las causas fundamentales de la deforestación. Las cifras de cambio de uso de suelo en el país señalan que la superficie agrícola ha seguido creciendo, incluso más rápidamente en los últimos años. Mientras que entre 1976 y 1993 se transformaban poco más de 179.000 hectáreas anualmente (en promedio), esa cifra alcanzó las 272.000 hectáreas entre 2002 y 2007, y entre 2007 y 2011 disminuyó a 100.000 hectáreas. En contraste, las áreas dedicadas a la ganadería y los pastizales cultivados o inducidos 
se han venido reduciendo de manera sobresaliente. De esta forma, la dispersión demográfica a través de la existencia de un gran número de localidades muy pequeñas se convierte en un factor de desequilibrio ambiental.

\section{La madera cobra venganza:}

\section{deforestación en la Península de}

\section{Yucatán en el siglo XXI}

Entre la población local de la Península de Yucatán existe la firme creencia de que "la madera cobra venganza" a raíz de experiencias de personas a las que les iba muy bien profesionalmente, que hicieron un patrimonio y decidieron invertirlo en el riesgoso sector de la explotación maderera. Son varias las historias de individuos que perdieron todo de esta forma, desde su fortuna hasta, incluso, su propia familia. Esta cuestión "kármica" se podría aplicar no solamente a nivel personal, sino también colectivo, ya que los efectos derivados de la deforestación y de la degradación forestal, como el incremento de emisiones de gases de efecto invernadero (GEI), han de repercutir en la población a nivel mundial, ya que esta es la causa principal del cambio climático.

La concentración de $\mathrm{CO}_{2}$ en la atmósfera ha pasado de tener aproximadamente 280 partes por millón (ppm) en la época preindustrial a 396 ppm en 2013. Hay que tener en cuenta que en $\operatorname{los} 800.000$ años de los que se cuenta con datos históricos, la concentración de este gas no había sido mayor a $300 \mathrm{ppm}$. Los procesos de deforestación y degradación favorecen que los ecosistemas forestales pierdan su condición de captores de $\mathrm{CO}_{2}$, contri- buyendo así al cambio climático. El informe del Grupo Intergubernamental de Expertos sobre el Cambio Climático (IPCC), presentado en 2007, reconoce que entre 1970 y 2004 las emisiones mundiales de $\mathrm{CO}_{2}$ y otros gases de efecto invernadero se han incrementado en un $70 \%$. De acuerdo con esta misma fuente, las emisiones asociadas al cambio de uso de suelo forestal fueron las terceras en importancia (17,4\%), después de las emitidas por los sectores de energía $(25,9 \%)$ y de la industria $(19,4 \%)$.

La Organización de las Naciones Unidas para la Agricultura y la Alimentación (FAO, por su sigla en inglés) estima que "las reservas de carbono en la biomasa forestal descendieron en 0.5 gigatoneladas (Gt) al año en el periodo 2000-2010, principalmente debido a la reducción de la superficie forestal total", lo que se tradujo en el incremento de emisiones de GEI.

Debemos tener en cuenta que los bosques tropicales contienen aproximadamente un $40 \%$ del carbono acumulado en la biomasa terrestre. Resulta claro que cualquier alteración de estos ecosistemas se reflejará en un cambio en el ciclo de carbono global y en un incremento de los impactos adversos. Esa es la razón por la cual los ojos del mundo entero están puestos en masas forestales como la selva localizada en la Península de Yucatán, ya que es uno de los últimos "pulmones" importantes que le quedan al planeta para poder detener el cambio climático.

Los países desarrollados reconocen la importancia de mitigar las emisiones por degradación y deforestación en los países en desarrollo, y son conscientes de que es más rentable económicamente actuar de manera temprana. Es por ello que, en 
el seno de la Convención Marco de las Naciones Unidas sobre el Cambio Climático, desde el 2010 se han ido celebrando sucesivas Conferencias de las Partes (COP). Además, hay que considerar que los bosques también contribuyen a atenuar el impacto de eventos hidrometeorológicos extremos (huracanes, sequías, ondas de calor o frío y tormentas, entre otros), aumentando así la resiliencia de la sociedad ante el cambio climático, además de generar múltiples beneficios como la conservación de la biodiversidad y la conectividad ecológica a nivel de paisaje, lo que permite la migración de especies.

La región tropical de la Península de Yucatán ha sufrido fuertes procesos de deforestación, tanto históricamente como en la actualidad, pues según datos de la Comisión Nacional Forestal (Conafor), de 1993 al 2002 los estados de Campeche y Yucatán fueron de los que más pérdidas de cobertura forestal registraron, con 310 y $230 \mathrm{~km} 2 /$ año respectivamente. Para el mismo periodo, en Quintana Roo se reportan $113 \mathrm{~km} 2 /$ año de pérdida neta de cobertura forestal, lo que equivale a una tasa de entre $0,3 \%$ y $0,4 \%$, que es la mitad de lo que se ha calculado para las otras dos entidades con las que comparte la península.

Sin embargo, para el periodo siguiente, de 2002 al 2013, con base en datos de Global Forest Change (GFC), se calcula un porcentaje neto de pérdida de cobertura forestal para Quintana Roo del 5,5\%, lo cual supera a Campeche $(5,1 \%)$ y a Yucatán $(4,2$ $\%$ ). Esta situación se refleja en el cálculo del porcentaje de pérdida de cobertura forestal neta por año, con -0,42 \%/año para Quintana Roo, - $0,39 \%$ / año para Campeche y $-0,32$ \%/año para Yucatán.

Teniendo en cuenta la superficie deforestada anual en la Península de Yucatán, tenemos un promedio de $800 \mathrm{~km}^{2}$ de pérdida neta en el periodo 2001 2013 de acuerdo con los datos de GFC, lo cual se traduce en que alrededor de un $60 \%$ de la deforestación nacional anual ocurrió precisamente dentro de esta península, donde tan solo en el año 2016 se perdieron $954 \mathrm{~km} 2$ de bosque y selva distribuidos de la siguiente forma:

- En Campeche se deforestaron $548 \mathrm{~km}^{2}$ de selva equivalentes al $22 \%$ del total deforestado a nivel nacional y más de la mitad de lo perdido en la península. La superficie deforestada pasó de 402 a $547 \mathrm{~km}^{2}$, un incremento del $36 \%$.

- En Yucatán se perdió el $23 \%$ de las selvas al pasar de 186 a $228 \mathrm{~km}^{2}$.

- En Quintana Roo, $368 \mathrm{~km}^{2}$ se perdieron en 2015 y $178 \mathrm{~km}^{2}$ en 2016, para un porcentaje de $-52 \%$, es decir que se deforestó menos en el último año.

Según cifras de GFC, de 2015 a 2016 México ha perdido $25,3 \mathrm{~km}^{2}$ de bosque y selva, la mayoría en Chiapas y en la Península de Yucatán, y la deforestación aumentó un $37 \%$ a nivel nacional.

Ellis, Romero y Hernández (2015) destacan cuatro causas principales y directas de deforestación en la Península de Yucatán para el periodo de 2001-2013:

- Expansión ganadera (50\% de la superficie deforestada) presente en todo el territorio.

- Agricultura de subsistencia (16\% de la superficie deforestada), también distribuida por toda la península.

- Agricultura mecanizada (13\% de la superficie deforestada), localizada en el norte de Campeche y la zona colindante de Yucatán. 
- Incendios forestales (10\% de la superficie deforestada), que se destacan en la zona norte y sur de Quintana Roo, así como en zonas próximas a Mérida.

\section{Revisión de la tasa de}

deforestación y usos de suelo en

el área bajo disputa territorial

\section{entre los municipios de Othón P. Blanco y Calakmul}

El área de estudio en este trabajo se ubica en la parte más meridional del sureste del territorio que comprende la República Mexicana, entre los paralelos $17^{\circ} 49^{\prime} 00^{\prime \prime}$ y $18^{\circ} 1^{\prime} 2^{\prime \prime}$, y los meridianos $89^{\circ} 09^{\prime} 07^{\prime \prime}$ y $89^{\circ} 24^{\prime} 53$ " (polígono amarillo en la Figura 1). Este espacio es el resultado de una disputa territorial derivada de la falta de acuerdo acerca del trazado de los límites territoriales entre los estados de Campeche, Yucatán y Quintana Roo, lo cual genera la indeterminación jurisdiccional de una franja de tierra de unos $10.500 \mathrm{~km}^{2}$ (polígono rojo en la misma Figura 1), que afecta, entre otros, al municipio campechano de Calakmul y a su vecino, Othón P. Blanco, perteneciente al Estado de Quintana Roo.

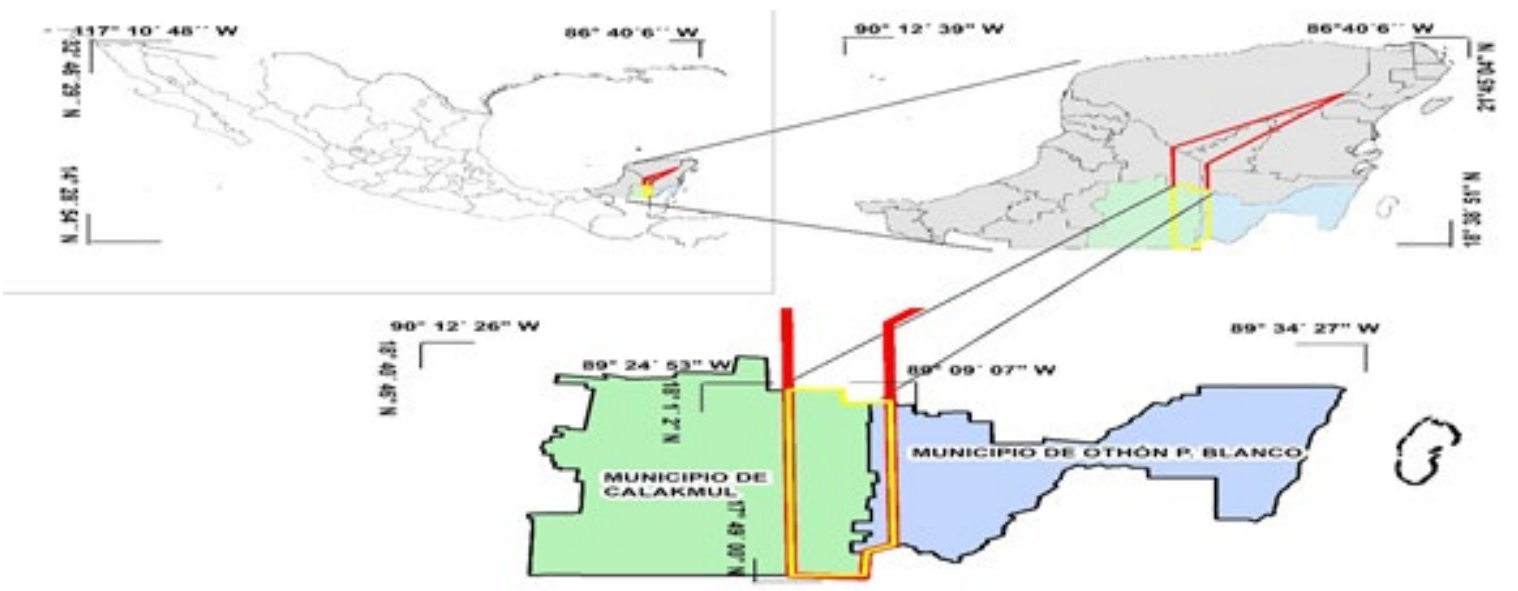

FIGURA 1.

Esquema de ubicación geográfica del área de estudio en el contexto nacional y estatal Fuente: Elaboración propia con base en Inegi (2016).

En el territorio en disputa, el tipo de vegetación que más abunda, con diferencia, es la selva, con un $77 \%$ de la superficie, lo que equivale a unos 5.981 $\mathrm{km}^{2}$. Este porcentaje tan elevado no es de extrañar si tenemos en cuenta que el $90 \%$ del territorio del Estado de Quintana Roo se conforma por estos bosques tropicales y que el $99 \%$ de la producción de maderas preciosas en el Estado de Quintana Roo se concentra en los municipios de Othón P. Blanco y Felipe Carrillo Puerto (Poder Ejecutivo del Estado de Quintana Roo, 2017). 
El tipo de selva es alta y mediana y existe la presencia de dolinas, conocidas localmente como aguadas o akalchés. Esta palabra de origen maya está formada por dos vocablos, akal, que significa pantano, y ché, que significa conjunto de árboles, por lo que hace referencia a un bajío, terreno bajo, pantano u hondonada donde se estancan las aguas. Estas depresiones son de origen kárstico, lo que favorece la infiltración de las aguas pluviales, por lo que tan solo se inundan durante la época de lluvias y son la única fuente de agua natural durante la época seca, que se alarga desde el mes de noviembre al de abril. En estos espacios se desarrollan comunidades de selva baja subperennifolia ${ }^{11}$, como palo de tinte (H. campechianum), sak cheechem (C. latifolia), muuk (Dalbergia glabra), chak ch'obenché (Erythrina standleyana) y chooch kitam (H. winzerlingii), entre otras. De acuerdo con Noriega-Trejo y Arteaga (citados en Comisión Nacional para el Conocimiento y Uso de la Biodiversidad [Conabio] y Secretaría de Medio Ambiente y Recursos Naturales de Campeche [Semarnatcam], 2016), los akalchés son ecosistemas donde habitan especies como el cocodrilo de pantano (Crocodylus moreletti), la tortuga pochitoque ( $\mathrm{Ki}$ nosternon creaseri), 129 especies de aves, el mono aullador (Alouatta pigra), el mono araña (Ateles geoffroyi), el jaguar (Panthera Onca) y el tapir (Tapirus bairdii).

La selva existente es cálido-húmeda, presente en toda la Península de Yucatán, salvo en la parte más noroeste. En concreto, en la colindancia de Quintana Roo, Campeche, Guatemala y Belice

11 Este tipo de selva se caracteriza porque sus especies pierden las hojas en época de seca y alcanzan alturas de entre 5 y 15 metros, con troncos de un diámetro no mayor a 50 centímetros. Se encuentra en terrenos arcillosos, profundos, con drenaje deficiente y está asociada con vegetación secundaria y agricultura normal. hay selva alta subdcaducifolia (Fragoso, Pereira, Frausto y Bautista, 2014) y subperennifolia, cuyas especies más representativas son: chicle (Manilkara zapota), caoba (Swietenia macrophylla), pukté (Bucida buceras) y ramón (Brosimum alicastrum), de acuerdo con Noriega-Trejo y Arteaga (citados en Conabio y Semarnatcam, 2016).

En cuanto a divisiones florísticas de México se refiere, toda la Península de Yucatán pertenece a la región Caribea (Conabio, 2015a). En el espacio geográfico objeto de estudio la selva tiene que convivir con áreas agrícolas, presentes en un $16 \%$ de su superficie $\left(1.293 \mathrm{~km}^{2}\right)$. Los pastizales apenas ocupan un $3 \%$ de la superficie $\left(242 \mathrm{~km}^{2}\right)$, las áreas agrícolas, un $2 \%\left(151 \mathrm{~km}^{2}\right)$, y otras categorías (áreas urbanas, cuerpos de agua y otros tipos de vegetación como el popal, de ambiente acuático), otro $2 \%\left(35 \mathrm{~km}^{2}\right)$ (Figura 2$)$.

En el área objeto de estudio hay que destacar que hay una media poblacional de 259 habitantes por cada una de las 33 comunidades existentes. Este poblamiento rural escaso y disperso puede traer consigo efectos depredadores en el ambiente, pues modifica el uso del suelo con graves consecuencias para la biodiversidad y el equilibrio de los ecosistemas. Inicialmente en estas selvas húmedas del sureste de México se establecieron grandes ranchos de cientos de hectáreas, pero más recientemente se adoptó un modelo de pequeñas unidades de producción a partir del proceso de colonización con ejidos y comunidades que practican ganadería y agricultura asociadas a la existencia de un alto número de pequeñas localidades no mayores a los 50 habitantes.

La producción agrícola en el territorio en disputa es considerada por la Conabio (2015b, 2015c, 2015 d y 2015 e) de crecimiento promedio anual 

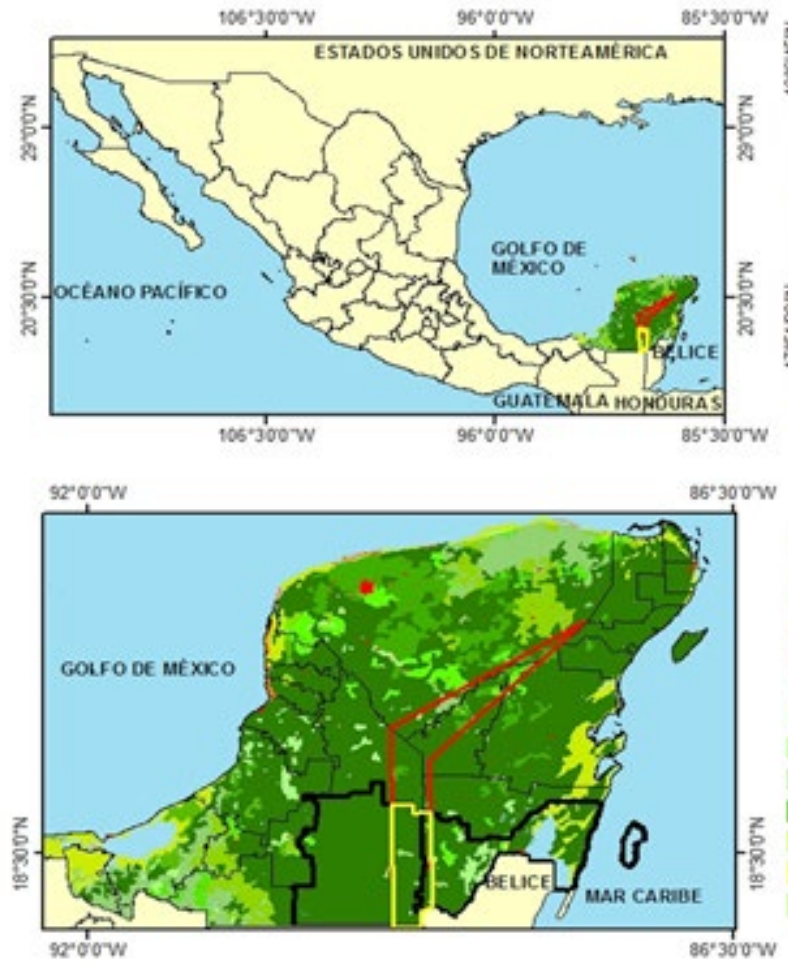

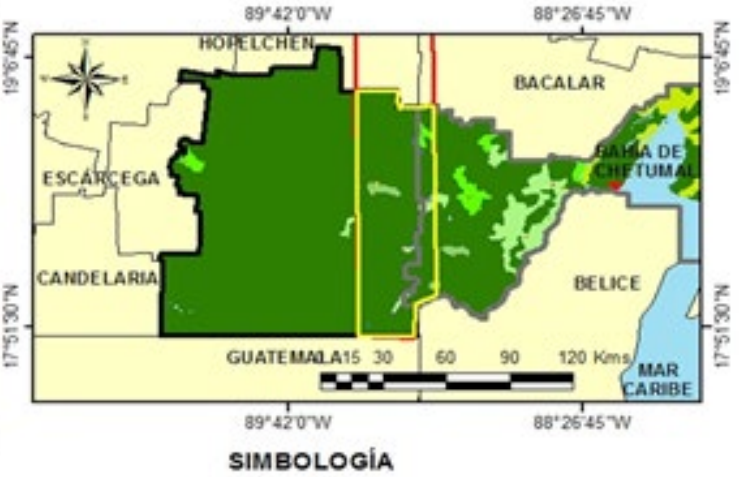

TPOS DE USOS DE SUELO Y VEGETACION

II AREAAGRICOLA

AREAMGRICOLAPASTIZAL

areaAgricolaselva

AREA SIN VEGETACIÓN

AREA URBAAVA

CUERPO DE AGUA

1 PASTIZAL

PASTIZN-SELVA

SELVA

selvaAREAMGRICOLA

SELVA.PASTIZAL.

OTROS TIPOS DE VEGETACIÓN

OTROS TIPOS DE VEGETACIÓN. PASTIZAL

REGIÓN DE ESTUDIO

$\square$ TERRITORIO EN DISPUTA

$\square$ MUNICIPIO DE OTHON P. ELANCO

MUNICIPIO DE CALAKGUL

contrexto

ESCALA NUMERICA: 12500.000 METADATOS

SISTEMADE COORDENADAS GEOGRAFICAS

DATUM: WGSPA

UNIDADES GRADOS

\section{FIGURA 2.}

Vegetación y tipos de usos de suelo en el territorio en disputa

Fuente: Elaboración propia con base en Inegi (2016).

constante en lo que se refiere a cultivos perennes de riego, que son aquellos cuyo ciclo vegetativo es mayor de un año; sin embargo, la tasa de crecimiento anual de las cosechas de dichos cultivos perennes baja una categoría, a estable. Aún decae una categoría más, a decremento constante, si atendemos la tasa de crecimiento promedio anual del precio medio rural o del valor de la producción de dichos cultivos.

Si tenemos en cuenta la producción de miel, en el periodo que va del 2007 al 2013 se puede observar que en el territorio en disputa existe un crecimiento constante, al igual que en su precio y en el valor de su producción (Conabio, 2015f, 2015g y 2015h). De hecho, la Península de Yucatán ocupa el tercer lugar a nivel mundial en exportación de miel, lo cual se debe no solo a la tradición heredada desde los tiempos prehispánicos de cuidar abejas, sino también a la exuberante flora apibotánica existente en la región, como la melífera y la polinífera.

Los productores reconocen que es una actividad redituable y complementaria de la economía campesina, pero falta capacitación para el manejo, créditos adecuados y facilidades para comercializar el producto orgánico obtenido. Esta actividad tiene grandes posibilidades económicas como alternativa para el desarrollo sustentable de la región (García y Pat, 2000).

A pesar de las limitaciones naturales para la producción, de la escasez de agua para consumo humano, del alto grado de marginalidad y de la 
lejanía de los mercados para sus productos agropecuarios, la colonización ha sido un factor decisivo para la deforestación de la selva (García y Pat, 2000).

Las investigaciones realizadas en este espacio geográfico coinciden en que en el periodo que va de 1988 al 2000 la tasa de deforestación estuvo entre $-0,3 \%$ y $-0,4 \%$, y señalan la expansión de la ganadería como la causa principal, mientras que los programas de desarrollo agrícola y los créditos y subsidios ganaderos se pueden considerar como factores secundarios en importancia.

En un estudio llevado a cabo por Rueda (2010), se encontró que en el periodo de 1984 a 1993 las tasas de deforestación fueron de casi $5 \%$ (fase de experimentación de desarrollo agrícola por parte del gobierno), mientras que esta tasa disminuyó a cerca de 0,5\% anual en el periodo 1993-2000. La región experimentó el crecimiento de la agricultura y la ganadería con cultivos como chile jalapeño en algunos ejidos grandes y antiguos y en ejidos pequeños cercanos a la carretera Escárcega-Chetumal.

Ellis et al. (2015) aseguran que se ha podido evidenciar, por medio de trabajo de campo, que en la parte poniente de esta región el principal cultivo es el maíz, localizado en terrenos cercanos a las localidades y próximos a vías de comunicación (como la carretera Escárcega-Chetumal), mientras que en la parte más oriental se aprecian potreros remontados, con cercas en mal estado y, en algunos casos, con pocas cabezas de ganado. En el sur del polígono analizado, en la frontera con Guatemala, refieren Ellis et al. (2015) que hay nuevos centros de población ejidal que culturalmente traen consigo las prácticas agropecuarias de potreros o pastizales.
El fenómeno de la deforestación se encuentra aparejado con caminos y brechas que han propiciado la alteración de la cubierta vegetal. Del periodo de 1900 a 1960 quedaron los caminos de los chicleros y el arrastre de las maderas preciosas hacia los tumbos y aserraderos de Zoh Laguna y San Antonio Soda. Posteriormente, a finales de los años sesenta, fue construida la principal vía de comunicación transpeninsular: la carretera Escárcega-Chetumal. La terminación de esta importante obra trajo consigo, en los años setenta, el poblamiento espontáneo a lo largo del camino, y con esto comenzó la apropiación reciente de la región. En los años ochenta se llevaron a cabo los trabajos formales del camino de terracería de Xpujil-Yohaltún-Chenes, que atraviesa el núcleo de la reserva, y en los noventa, el camino de terracería Xpujil-frontera Guatemala. Actualmente ambos se encuentran pavimentados.

García y Pat (2000) reconocen que "esta infraestructura vial pudo contribuir, en parte, a disminuir la marginalidad de las comunidades, pero ha tenido también una repercusión colateral: el surgimiento de nuevas localidades humanas que representan mayor presión demográfica hacia los recursos y mayor expansión de las fronteras agropecuarias" (p. 223).

En cuanto a la dinámica de cambios en el uso de suelo en la región de estudio, Ellis et al. (2015) pudieron observar que los acahuales jóvenes pasan a ser nuevamente tierra de cultivo de maíz, tras cinco o siete años de barbecho, mediante el sistema de roza-tumba y quema. Tras dos o tres años destinados a la agricultura, durante los cuales el suelo pierde paulatinamente su fertilidad debido a su explotación consecutiva, los predios se dejan en descanso otra vez y pueden emplearse como potreros. Los ejidatarios o personas dedicadas a dicha actividad 
mencionan que períodos más cortos de barbecho producen menor cantidad y mala calidad de maíz. Lo idóneo serían periodos de recuperación de 15 a 20 años, necesarios para recobrar la fertilidad del suelo por medio de la materia orgánica que se incorpora durante el proceso natural de crecimiento de la selva.

En suma, los asentamientos humanos están incidiendo sobre los recursos naturales de la zona objeto de estudio, lo que se traduce en cambios de uso del suelo con la intención de realizar actividades agropecuarias. El incremento de este tipo de superficies explica la expansión de la frontera agrícola y la consolidación de la apropiación del suelo.

\section{6. ¿Qué se está haciendo para revertir la situación?}

Se estima que los bosques y selvas mexicanos se encuentran entre los primeros 24 del mundo con mayor volumen de existencias de carbono en la biomasa forestal viva. México reconoce lo importante que es conservar, manejar y restaurar los ecosistemas forestales y sus invaluables bienes y servicios ambientales. Para ello, desde hace al menos un par de décadas, se están llevando a cabo políticas públicas para lograr que las comunidades humanas que los habitan obtengan mejores oportunidades de bienestar a través de su manejo sustentable. Estos esfuerzos son fundamentales para luchar contra el fenómeno del cambio climático.

Para atender la problemática de la disminución de las superficies forestales, frenar el proceso de deterioro de la vegetación forestal y revertir la tendencia del cambio de uso del suelo forestal y la conse- cuente pérdida de vegetación forestal en la región de la Península de Yucatán, la Conafor, en el 2012, implementó el Programa Especial para la Conservación, Restauración y el Manejo Sustentable de los Recursos Forestales de la Península de Yucatán y el Programa Proárbol, que consistían en asignar y otorgar apoyos a los beneficiarios que cumplieran con los requisitos exigidos en las reglas de operación y los lineamientos correspondientes.

El manejo sustentable del medio ambiente mide la capacidad de las entidades federativas para aprovechar el potencial de los recursos naturales sin poner en riesgo su sustentabilidad en el mediano y largo plazo. En este sentido, las Áreas Naturales Protegidas juegan un papel de gran relevancia, pues ofrecen las particularidades que permiten conservar los ecosistemas al tiempo que asumen el factor social como aspecto clave para el cuidado y el uso sostenible de los recursos en beneficio de la sociedad y del entorno natural por igual. Concretamente, en la Región Península de Yucatán hay 24 áreas protegidas que se extienden a lo largo de 28.000 kilómetros cuadrados distribuidos de la siguiente forma:

- Actualmente, el 12\% del territorio del Estado de Yucatán se encuentra bajo condiciones de protección ecológica, lo que equivale a unos $5.200 \mathrm{~km}^{2}$ (Poder Ejecutivo del Estado de Yucatán, 2013).

- Quintana Roo cuenta con $6.317 \mathrm{~km}^{2}$ de área protegida, lo que representa el $12 \%$ de su superficie territorial y lo sitúa en novena posición a nivel nacional desde los años 2012 y 2014, de acuerdo con su Plan Estatal de Desarrollo 2016-2022. 
- Cerca del $40 \%$ del territorio campechano se encuentra bajo algún régimen de protección ecológica. Existen cuatro Áreas Naturales Protegidas de carácter federal, dos administradas por el Estado de Campeche y una de administración municipal. En total, estas áreas abarcan $22.324 \mathrm{~km}^{2}$, de acuerdo con el Plan Estatal de Desarrollo de Campeche para el periodo 2015-2021 (Poder Ejecutivo del Estado de Campeche, 2015).

De entre todas esas Áreas Naturales Protegidas, tres conviven con el territorio en disputa señalado en este estudio, Bala'an K'aax (1.284 km²), la Laguna de Chichankanab $\left(1.999 \mathrm{~km}^{2}\right)$ y la Reserva de la Biosfera de Calakmul. En particular, ésta última destaca por ser el área protegida con mayor superficie de selvas tropicales de México $\left(7.232 \mathrm{~km}^{2}\right)$.

$\mathrm{Su}$ valor biológico a escala regional es de gran importancia porque posibilita la continuidad biológica con las reservas y áreas protegidas "vecinas" en Guatemala y Belice, con las que aún mantiene continuidad forestal; la superficie ininterrumpida de selva asciende a más de $30.000 \mathrm{~km}^{2}$. Sin embargo, en esta gran selva de la región maya existe una creciente presión antrópica por el cambio de uso del suelo, debido a la expansión de la frontera agropecuaria, lo que genera un proceso de fragmentación y reducción de la selva (García y Pat, 2000, p. 214).

Desde el año 2013, los tres gobiernos de las entidades peninsulares, en coordinación con el Banco Mundial, han trabajado para integrar un Fondo de Cambio Climático de la Península de Yucatán, con la finalidad de generar mecanismos de financia- miento y distribución de beneficios. En tres años se han generado las condiciones institucionales, de planeación y de consensos para comenzar la ejecución conforme a la normatividad aplicable.

En diciembre del 2016 se aprobó la Iniciativa de Reducción de Emisiones para México, conocida como IRE, en la quinta reunión del Fondo Cooperativo de Carbono Forestal (FCPF). En dicha iniciativa se establece el compromiso de reducir las emisiones de carbono relacionadas con el uso del suelo en cinco estados del país, incluido Quintana Roo, bajo un esquema de pago por resultados. Para ello hay que promover acuerdos con los usuarios de la tierra y alinear la intervención gubernamental con este objetivo.

Actualmente la Estrategia Nacional para la Reducción de Emisiones por Deforestación y Degradación (ENAREDD+) contribuye a la mitigación de GEI planteando políticas, medidas y acciones que deben ser incorporadas en instrumentos de planeación que promuevan la reducción de la deforestación y la conservación, así como el manejo y la restauración de los recursos forestales. Son igual de importantes las Estrategias Estatales REDD $+($ EEREDD +$)$ para las actividades en los estados que promuevan un desarrollo territorial integrado.

Esta estrategia responde a la iniciativa internacional iniciada en Bali en 2007 en la Convención Marco de las Naciones Unidas para el Cambio Climático (CMNUCC), con el objetivo de incentivar el manejo forestal sustentable y así reducir las presiones sobre los ecosistemas forestales, derivadas de otras actividades y de circunstancias económicas. Para ello se pretende contar con la colaboración de múltiples actores sociales y de las instituciones públicas que intervienen en el 
desarrollo territorial. Muestra de ello es que existe un Comité para la Creación del Fondo de Acción Climática de la Península de Yucatán, en el que participa personal de los tres estados peninsulares, de la organización México-REDD + y de la banca comercial.

Ellis et al. (2015) proponen que las zonas de baja deforestación con potencial de conservación de selvas deben ser integradas a estrategias como esta y a programas institucionales, como el de Áreas Nacionales Protegidas o el de regiones de "cero deforestación"12. Por otro lado, en las zonas agropecuarias con alta amenaza de deforestación, coinciden en que habría que dar incentivos para la reforestación e integración de árboles.

La ENAREDD+ se está desarrollando en tres fases diferentes:

1. Preparación y difusión de la estrategia, desarrollo de las capacidades institucionales, monitoreo y verificación, así como realización de actividades en áreas de acción temprana. Modificación, diseño y alineación de los programas, políticas públicas y marco legal hacia modelos transversales que permitan un manejo sustentable de ecosistemas y paisajes forestales.

2. Evaluación, con indicadores medibles, de las políticas y acciones adoptadas a corto plazo.

12 La Conafor está diseñando estrategias, políticas, medidas y acciones para lograr el objetivo de alcanzar una tasa de $0 \%$ de pérdida de carbono en los ecosistemas originales con un horizonte en el 2020. De esa forma se cumpliría con una de las metas planteadas en la Ley General de Cambio Climático (LGCC) y en la Estrategia Nacional de Cambio Climático.
3. Finalmente, se pretende alcanzar los requisitos para acceder al mecanismo REDD + y poder recibir incentivos. Esto incluye contar con un sistema nacional de monitoreo, reporte y verificación (MRV) ya en operación (Conafor, 2014).

En el marco de las acciones tempranas REDD + y de la Conafor, se concluyó la $1^{\text {a }}$ etapa del Programa Especial de la Península de Yucatán y se obtuvieron recursos por 12,5 millones de pesos para apoyar a comunidades, los ejidos y los dueños de tierras, lo cual contribuye a las acciones tempranas de preparación e implementación del suelo (Plan de Desarrollo del Estado de Quintana Roo, 20162022).

\section{Conclusiones}

El paso de la explotación forestal extractiva a la sustentable aún se sitúa en un contexto en que la geopolítica es determinante bajo el enfoque de la expansión de las fronteras interiores, que reconoce que condiciones geográficas, políticas, sociales e históricas dentro del espacio geográfico de los Estados pueden limitar el desarrollo de regiones e, incluso, aislarlas del centro de la nación. Es indudable que la riqueza de la selva es el atractivo que, de manera constante, podemos encontrar en los diversos intereses exhibidos por quienes lograron, intentaron o aún intentan establecer su soberanía en la región. Desde el siglo XVII, el colonialismo europeo disputaba tanto el control efectivo como el dominio jurídico de la zona para la explotación del palo de tinte. España e Inglaterra lucharon por asegurar el ejercicio de la soberanía, la posesión y la propiedad de estas tierras. 
Como se mencionó, a finales del siglo XIX la desmesurada explotación extractiva del bosque, a cargo de los concesionarios y de las empresas forestales, aparte del beneficio económico que se perseguía, también significó una táctica del gobierno para ocupar e impulsar la colonización del territorio en manos de los mayas, quienes desde última mitad del siglo XIX, y aún entrado el XX, combatieron para conservar la selva que les servía de refugio, hogar, recinto sagrado y que les daba tantas cosas para su vida cotidiana. Era necesario ganar la guerra a los indígenas rebeldes para poder definir y controlar la frontera con Belice y, además, aprovechar las riquezas que el gobierno de Yucatán no había podido mantener bajo su dominio.

La colonización humana que se ha estado llevando a cabo en las últimas décadas en zonas selváticas de la Península de Yucatán conlleva la sustitución de las comunidades vegetales primitivas por sistemas agrícolas y pecuarios. A través del transcurso de los ciclos agrícolas y la escasa disposición de capacidades tecnológicas de los pobladores, se va ampliando paulatinamente la frontera agropecuaria mediante el reemplazo y la fragmentación de la selva.

Varias son las causas que han propiciado el cambio de uso de suelo del forestal al agropecuario. Por un lado, está la oferta de subsidios gubernamentales que fomentan el uso no sustentable de los recursos naturales y que suelen ser más beneficiosos que los subsidios forestales. Por otro lado, las tierras boscosas, en comunidades de alta marginación y cuya producción es principalmente para el autoconsumo, se convierten en nuevas parcelas para el cultivo, lo que genera una deforestación paulatina. Adicionalmente, la tala ilegal, la recolección excesiva de leña y la ganadería extensiva también contribuyen a la deforestación y a la degradación forestal. Tampoco se han de olvidar los incendios forestales como otro foco de atención especial en este asunto o el cambio de uso del suelo en zonas turísticas de Quintana Roo.

Una importante parte de la población que habita en el medio rural y depende de los ecosistemas forestales son comunidades con altos índices de pobreza y marginación. A pesar de esto, algunas comunidades, como Noh Bec y Tres Garantías, han alcanzado una gran experiencia en el manejo activo de sus bosques, que incluye una progresiva incorporación de superficies de manejo a esquemas de certificación.

En concreto, en el área de estudio delimitada en este trabajo, inserta en la Península de Yucatán, los espacios productivos de las comunidades están ocupados por cuatro actividades: la explotación ganadera impulsada en los años ochenta con apoyo de programas públicos en forma de potreros, la producción de maíz para autoconsumo mediante el uso de la técnica agrícola de roza, tumba y quema, el cultivo comercial del chile jalapeño y la práctica de la apicultura.

En la actualidad, debido a la deforestación ocasionada por la tala inmoderada y por los efectos de los fenómenos naturales y de los incendios, resulta que, al contrario de lo transcurrido en épocas anteriores, ahora se considera la conservación del patrimonio forestal como un asunto de seguridad nacional.

En México se deberían hacer esfuerzos para reducir la deforestación y la degradación de los bosques por medio de un desarrollo rural sustentable. Se considera que la conservación y el desarrollo son compatibles si se coloca la sustentabilidad 
como principio de actuación. Los elementos centrales de las actividades que se deberían llevar a cabo incluirían la creación de capacidades para la gestión territorial, la consolidación de programas forestales regionales, la innovación y la alineación de políticas y del marco legal, la gestión participativa, el monitoreo y la evaluación.

Los estados de Quintana Roo y Campeche se proponen como prioridad, en sus respectivos Planes de Desarrollo, el crecimiento sustentable y la preservación del patrimonio natural. Sin embargo, la tendencia actual en esta entidad federativa, es decir, Quintana Roo, es continuar incrementando la superficie deforestada, aunque a un ritmo más reducido que el de años anteriores.

Con el apoyo del Programa de las Naciones Unidas para el Desarrollo y con la participación de los estados de Campeche, Yucatán y Quintana Roo se concluyó la hoja de ruta de la estrategia regional de adaptación al cambio climático, encaminada a reducir y detener la deforestación y la degradación forestal, la cual es única en su tipo por ser la primera región del país en la que se trabaja conjuntamente.

Los resultados de estas políticas públicas ya se están obteniendo. La deforestación ha disminuido en la última década en la Península de Yucatán. Según el último reporte de la FAO sobre los recursos forestales del mundo, se confirma una baja en la tasa de deforestación en el territorio mexicano, de $-0,3 \%$ (1.900 km²/año) entre 1990 y 2000 a $-0,2 \%$
(1.340 km²/año) entre 2000 y 2010, y, finalmente, baja a $-0,1 \%$ (920 km²/año) entre el 2010 y 2015 . Esto tiene su reflejo en la caída de emisiones de carbono y contrasta con las cifras de GFC, según las cuales de 2015 a 2016 México ha perdido 2.530 $\mathrm{km}^{2}$ de bosque y selva, la mayoría en Chiapas y en la Península de Yucatán, y la deforestación aumentó en un $37 \%$ a nivel nacional.

Lo cierto es que las tendencias de deforestación aún persisten en zonas de la Península de Yucatán, y requieren de medidas y estrategias más puntuales y más exitosas para poder seguir reduciendo las emisiones por deforestación y degradación en dicho territorio.

La propuesta de solución que aquí se presenta es la posibilidad de la asociación de los municipios de Othón P. Blanco y Calakmul con fines de gestión ambiental. Esta figura les permitiría involucrarse dentro de las tareas del Sistema Nacional de Cambio Climático (SINACC), el cual funge como un mecanismo permanente de comunicación, colaboración, coordinación y concertación sobre la política nacional de cambio climático, de acuerdo con lo dispuesto en los artículos 38 al 44 de la Ley General de Cambio Climático (LGCC).

El desarrollo y el continuo fortalecimiento de este marco institucional permitirá la consecución efectiva de los distintos objetivos y metas plateadas en la LGCC, así como con los compromisos internacionales en la materia por parte de México. 


\section{Referencias}

Álvarez, P. y Herrera, R. (2014). La dispersión de la población y la pérdida de biodiversidad. En J. L. Ávila, H. Hernández y J. Narro (coords.), Cambio demográfico y desarrollo de México (pp. 289-315). México, D. F.: Universidad Nacional Autónoma de México.

Amor-Conde, D., Colchero, F., Rivera, A., Manterola, C. (2005). Deforestación de 1978 a 2000 para el sur de Campeche y Quintana Roo [escala 1:250.000]. México: Unidos por la Conservación A.C.

Bracamonte y Sosa, P. (2000). La jurisdicción cuestionada y el despojo agrario en el Yucatán del siglo XIX. Revista Mexicana del Caribe, 10(5), 150-179.

Comisión Nacional para el Conocimiento y Uso de la Biodiversidad (Conabio) (2015a). Divisiones florísticas de México. En J. Rzedowski, Vegetación de México. México: Conabio.

Comisión Nacional para el Conocimiento y Uso de la Biodiversidad (Conabio) (2015b). Tasa de crecimiento promedio anual de la producción de los cultivos perennes de riego por municipio, 2003 a 2013, escala 1:25.0000. México, D. F.: Catálogo de metadatos Conabio.

Comisión Nacional para el Conocimiento y Uso de la Biodiversidad (Conabio). (2015c). Tasa de crecimiento promedio anual de las cosechas perennes de riego por municipio, 2003 a 2013, escala 1:25.0000. México, D. F.: Catálogo de metadatos Conabio.

Comisión Nacional para el Conocimiento y Uso de la Biodiversidad (Conabio). (2015d). Tasa de crecimiento promedio anual del precio medio rural de los cultivos perennes de riego por municipio, 2003 a 2013, escala 1:25.0000. México, D. F.: Catálogo de metadatos Conabio.

Comisión Nacional para el Conocimiento y Uso de la Biodiversidad (Conabio). (2015e). Tasa de crecimiento promedio anual del valor de la producción de los cultivos perennes de riego por municipio, 2003 a 2013, escala 1:25.0000. México, D. F.: Catálogo de metadatos Conabio.

Comisión Nacional para el Conocimiento y Uso de la Biodiversidad (Conabio). (2015f). Tasa de crecimiento promedio anual de la producción de miel por municipio, 2007 a 2013, escala 1:25.0000. México, D. F.: Catálogo de metadatos Conabio.

Comisión Nacional para el Conocimiento y Uso de la Biodiversidad (Conabio). (2015g). Tasa de crecimiento promedio anual del precio de la miel por municipio, 2007 a 2013, escala 1:250000. México, D. F.: Catálogo de metadatos Conabio.

Comisión Nacional para el Conocimiento y Uso de la Biodiversidad (Conabio). (2015h). Tasa de crecimiento promedio anual del valor de la producción de miel por municipio, 2007 a 2013, escala 1:250000. México, D. F.: Catálogo de metadatos Conabio. 
Comisión Nacional para el Conocimiento y Uso de la Biodiversidad (Conabio) y Secretaría de Medio Ambiente y Recursos Naturales de Campeche (Semarnatcam). (2016). Estrategia para la conservación y el uso sustentable de la biodiversidad en el Estado de Campeche. México: Conabio.

Comisión Nacional Forestal (Conafor). (2014). Estrategia Nacional para REDD+. Recuperado de http://www.enaredd.gob.mx/wp-content/uploads/2015/08/ Estrategia-Nacional-para-REDD.pdf.

Consejo Nacional de Población (Conapo). (2012). Índice de marginación por localidad 2010. Recuperado de http://www.conapo.gob.mx/es/CONAPO/Datos_Abiertos del_Indice_de_Marginacion

Consejo Nacional de Evaluación de la Política de Desarrollo Social (Coneval). (2010). Indicadores de pobreza extrema y carencia por acceso a la alimentación por municipio 2010. Recuperado de https://www.coneval.org.mx/Medicion/MP/Paginas/ Medicion-de-la-pobreza-municipal-2010.aspx

Ellis, E. A., Romero Montero, A. y Hernández Gómez, I. U. (2015). Evaluación y mapeo de los determinantes de deforestación en la Península Yucatán. México, D. F.: Agencia de los Estados Unidos para el Desarrollo Internacional (USAID), The Nature Conservancy (TNC), Alianza México REDD+.

Ferré, R. (2001). La apropiación del espacio geográfico de la Península de Yucatán: El caso Campeche. En G. Arruda, D. Velázquez y G. Zuppa (Orgs.), Natureza na América Latina: apropriações e representações (pp. 218-227). Londrina: Ed. UEL.

Fragoso, P., Pereira, A., Frausto, O. y Bautista, F. (2014). Relación entre la geodiversidad de Quintana Roo y su biodiversidad. Quivera, 16(1), 97-125.

García, G. y Pat, J. M. (2000). Apropiación del espacio y la colonización en la Reserva en la Biosfera de Calakmul, Campeche, México. Revista Mexicana del Caribe, 5(10), 212-231.

Global Forest Change (2018). Where did tree cover loss occur in Mexico. Recuperado de http:// www.globalforestwatch.org

Grupo Intergubernamental de Expertos sobre el Cambio Climático (IPCC). (2007). Cambio climático 2007. Informe de síntesis. Contribución de los Grupos de trabajo I, II y III al Cuarto Informe de evaluación del Grupo Intergubernamental de Expertos sobre el Cambio Climático [Equipo de redacción principal: Pachauri, R. K. y Reisinger, A. (directores de la publicación)]. Ginebra, Suiza: IPCC. Recuperado de https://www.ipcc.ch/site/assets/uploads/2018/02/ar4_syr_sp.pdf

Higuera, A. (1997). Quintana Roo entre tiempos. Política, poblamiento y explotación forestal, 1872-1925. Chetumal: Universidad de Quintana Roo e Instituto Quintanarroense de la Cultura.

Hoy, C. (1998). Breve historia de Quintana Roo. Chetumal: Gobierno de Quintana Roo.

Instituto Nacional de Estadística y Geografía (Inegi). (2016). Anuario estadístico y geográfico de los Estados Unidos Mexicanos 2016. Recuperado de http://internet. contenidos.inegi.org.mx/contenidos/productos/prod_serv/contenidos/espanol/ bvinegi/productos/nueva_estruc/AEGEUM_2016/702825087340.pdf 
Laguna, M. (2005). Las desigualdades territoriales y el desarrollo del Estado de Quintana Roo, México. (Tesis doctoral). Universidad de La Habana, Cuba.

Leriche, L. F. (2001). Colonización y globalización, cuatro siglos de apropiación territorial: el caso de la Isla del Carmen en el Golfo de México. En G. Arruda, D. Velázquez y G. Zuppa (orgs.), Natureza na América Latina: apropriações e representações (pp. 256-257). Londrina: UEL.

Macías, G. A. (Coord.). (2004). El vacío imaginario. Geopolítica de la ocupación territorial en el Caribe oriental mexicano. México D. F.: Ciesas, H. Congreso del Estado de Quintana Roo.

Pino, E. (1997). Calakmul: una deuda histórica comienza a saldarse. En Calakmul: volver al sur (pp. 193-216). Campeche: Gobierno del Estado Libre y Soberano.

Poder Ejecutivo del Estado de Campeche (noviembre de 2015). Plan Estatal de Desarrollo del Estado de Campeche 2015-2021. Campeche: Comité de Planeación para el Desarrollo del Estado. Recuperado de http://comunicacampeche.com.mx/ PED20152021.pdf

Poder Ejecutivo del Estado de Quintana Roo (25 de enero de 2017). Plan Estatal de Desarrollo del Estado de Quintana Roo 2016-2022. Periódico Oficial del Estado de Quintana Roo, t. I, no 11 extraordinario. Novena época. Recuperado de https://bit. ly/2OgWMdB

Poder Ejecutivo del Estado de Yucatán (marzo de 2013). Plan Estatal de Desarrollo del Estado de Yucatán 2012-2018. Recuperado de http://sds.yucatan.gob.mx/politicaambiental/documentos/PED_2012_2018_Yucatan.pdf

Quintal, J. y Chan, J. (2011). La participación política en Quintana Roo. Origen y Desarrollo (1902-1981). Chetumal, México: IEQROO.

Ramayo, T. (1997). Estrategia política regional: Campeche y Quintana Roo 1934-1940. En Calakmul: volver al sur (pp. 131-142). Campeche: Gobierno del Estado Libre y Soberano.

Ramos, M. (1999). La bonanza del chicle en la frontera caribe de México. Indígenas y empresarios, 1918-1930. Revista Mexicana del Caribe, 7(4), 172-193.

Rueda, X. (2010). Understanding deforestation in the southern Yucatán: insights from a subregional, multi-temporal analysis. Regional Environmental Change, 10(3), 175-189.

Sobrino, J., Garrocho, C., Graizbord, B., Brambila, C., y Aguilar, A. G. (2015). Ciudades sostenibles en México: una propuesta conceptual y operativa. México: Producción Creativa.

Velázquez, D. (2001). Naturaleza e interpretación en la Península de Yucatán: el bosque tropical (1939-1999). En G. Arruda, D. Velázquez y G. Zuppa (orgs.), Natureza na Amŭrica Latina: apropriações e representações (pp. 167-188). Londrina: UEL. 